\title{
Consistent, Durable, and Safe Memory Management for Byte-addressable Non Volatile Main Memory
}

\author{
Iulian Moraru, David G. Andersen, Michael Kaminsky ${ }^{\circ}$, Niraj Tolia ${ }^{\ddagger *}$, \\ Parthasarathy Ranganathan ${ }^{\dagger *}$, Nathan Binkert ${ }^{\natural *}$ \\ Carnegie Mellon University, Intel Labs ${ }^{\circ}$, Maginatics $^{\ddagger}$, Google ${ }^{\dagger}$, Amiato $^{\natural}$
}

\begin{abstract}
This paper presents three building blocks for enabling the efficient and safe design of persistent data stores for emerging non-volatile memory technologies. Taking the fullest advantage of the low latency and high bandwidths of emerging memories such as phase change memory $(\mathrm{PCM})$, spin torque, and memristor necessitates a serious look at placing these persistent storage technologies on the main memory bus. Doing so, however, introduces critical challenges of not sacrificing the data reliability and consistency that users demand from storage. This paper introduces techniques for (1) robust wear-aware memory allocation, (2) preventing of erroneous writes, and (3) consistency-preserving updates that are cacheefficient. We show through our evaluation that these techniques are efficiently implementable and effective by demonstrating a $\mathrm{B}+$-tree implementation modified to make full use of our toolkit.
\end{abstract}

\section{Introduction}

For decades, with a few niche, expensive exceptions, fast memory has been volatile, and persistent storage has been slow. The advent of non-volatile, byte-addressable memories may force an overhaul of program and operating system structure comparable to that required to deal with multicore. There are multiple competing technologies (Phase Change Memory, Spin Torque Transfer, memristor) but the end result is the same: a non-volatile,

${ }^{*}$ This work was done while the author was at HP Labs.

Permission to make digital or hard copies of part or all of this work for personal or classroom use is granted without fee provided that copies are not made or distributed for profit or commercial advantage and that copies bear this notice and the full citation on the first page. Copyrights for third-party components of this work must be honored. For all other uses, contact the Owner/Author.

Copyright is held by the Owner/Author(s).

TRIOS'13, Nov 03 - November 03 2013, Farmington, USA.

ACM 978-1-4503-2463-2/13/11.

http://dx.doi.org/10.1145/2524211.2524216 byte-addressable memory (we refer to it as NVRAM) that, when placed directly on the memory bus, will be almost as fast to access as DRAM.

We expect that uses for fast persistent memory will range from easy to program but low-performance (e.g., using it as a replacement for Flash memory), to complex and challenging setups meant to benefit performancecritical applications. In this paper we focus on the latter category. For reasons outlined in Section 2.2, we argue that performance-critical applications will require placing fast non-volatile memory on the memory bus alongside DRAM, and accessing both through CPU loads and stores (see diagram below):

\begin{tabular}{|c|}
\hline \multicolumn{2}{|c|}{ Applications } \\
\hline OS $\quad \begin{array}{c}\text { Persistence } \\
\text { Library }\end{array}$ \\
\hline $\begin{array}{c}\text { Main memory } \\
\text { DRAM + NVRAM }\end{array}$ \\
\hline
\end{tabular}

Furthermore, we argue that harnessing this performance can best be achieved through combined hardwaresoftware designs-as opposed to exclusively hardware or exclusively software solutions.

The fundamental challenge when using non-volatile main memory is to attain high throughput while providing strong durability guarantees. Many more things can go wrong when using the main memory bus to access persistent storage. One challenge is wear-out: some non-volatile memories degrade with writes. (e.g. excessive writes to a phase change memory cell can rapidly destroy it). A second challenge comes from the interface presented to the application writer with respect to durability and safety. Today's storage interfaces are narrow and explicit: Writes to storage can only affect data; only the kernel can change filesystem metadata. A write () makes data durable (in theory, depending on the implementation), and writes to hard disks typically succeed or fail atomically on a page or sector granularity, enforced by hardware sector checksums. With non-volatile memory, however, erroneous writes can affect persistent data or metadata. The order of writes is unknown unless special attention is taken to flush writes, due to interactions with processor caches.

Decades of experience with filesystems and databases 
have shown that developing safe and fast persistent data stores is challenging, and we do not believe that emerging memory technologies will change this. This paper describes three building blocks to ease the task of the persistent data structure designer (née filesystem or database designer), who develops libraries that would be linked against by application developers. These building blocks help address some of the primary potential sources of data loss and corruption when using non-volatile memory, while imposing minimal performance overhead. All three are designed across hardware-software boundaries, leveraging minimal hardware support: functionality already widely available but used sparingly, functionality previously proposed by other researchers, and new functionality that we propose in this paper:

Wear-aware memory allocation that is robust to erroneous writes. Hardware wear leveling [30, 31, 36] is necessary, but does not provide a complete solution. In particular, coping with frequent writes to the same or neighboring locations imposes overheads when writing (e.g., higher than 100\% [31]), as the hardware constantly shifts the data to new locations. Maintaining maximal throughput therefore requires software to avoid these pathological patterns. Traditional memory allocators do not avoid these pitfalls. At the same time, corruption of memory allocator metadata can lead to permanent data loss and unrecoverable memory leaks. To address these problems, we introduce a new memory allocator that complements hardware wear leveling for NVRAM. Our allocator prevents the frequent reuse of memory locations, and stores its frequently-changing metadata in DRAM. It adds checksums to detect and recover from metadata corruption.

A low overhead mechanism for containing erroneous writes. With DRAM, the consequence of bugs or memory corruption is typically an application crash. With NVRAM, however, erroneous writes can cause permanent data loss. The larger the persistent memory area exposed to the application for direct access, the higher the risk of corruption through erroneous writes. Using virtual memory protection to contain this threat has been proposed previously in the context of databases [38] and reliable file system caches [8]. In this paper, we show how to implement a similar scheme in a way that avoids the overhead of system calls and mode switches. This scheme complements the protection provided by our robust memory allocator: VM protection prevents "longrange" erroneous writes to locations that clearly should not be written to; the robust allocator protects against farreaching consequences from "short-range" off-by-one errors or buffer overflows.

Cache-efficient, consistency-preserving updates. Effective CPU caching is critical for speed. However, en-

\begin{tabular}{lcc} 
Parameter & PCM & DRAM \\
\hline Read Latency & $50 \mathrm{~ns}$ & $20-50 \mathrm{~ns}$ \\
Write Latency & $150 \mathrm{~ns}$ & $20-50 \mathrm{~ns}$ \\
Read Bandwidth & $1 \mathrm{~GB} / \mathrm{s} /$ die & $2 \mathrm{~GB} / \mathrm{s} / \mathrm{die}$ \\
Write Bandwidth & $200 \mathrm{MB} / \mathrm{s} /$ die & $2 \mathrm{~GB} / \mathrm{s} / \mathrm{die}$ \\
Write cycles & $10^{8}$ cycles & $\infty$
\end{tabular}

Table 1: Predicted PCM and DRAM characteristics $[1,3,28,29]$.

suring the consistency of persistent data in the face of application crashes and power failures requires careful control over the order of writes to persistent memory. Today's CPUs provide only coarse controls, e.g., by marking memory non-cacheable or by flushing the cache, but these methods impose high costs. We propose a novel mechanism for implementing consistency-preserving updates without sacrificing performance: make applications aware of where their data is at any time-in cache or in persistent memory. Our solution requires application support, as well as lightweight hardware modifications.

\section{Background and Assumptions}

This section briefly overviews non-volatile, byte addressable memories and enumerates the assumptions we make about future system support for these memories.

\section{$2.1 \quad$ Non-Volatile RAM}

Three emerging technologies hope to provide fast, persistent, byte addressable memories: phase change, memristor, and spin torque transfer. We collectively refer to these memories as non-volatile RAM (NVRAM). We focus primarily on phase-change memory, because it is now becoming commercially available and is better characterized, to understand how these memories differ from conventional DRAM.

Phase Change Memory (PCM) is a non-volatile, random-access memory that stores bits by heating a nanoscale piece of chalcogenide glass and allowing it to cool into either a crystalline (1) or an amorphous state (0), each of which has a different electrical resistance. Heating is performed by injecting current through the memory cell. Several of PCM's most important characteristics can be derived from this method of operation: programmable at byte granularity, fast reads but limited write bandwidth, wear-out.

Table 1 presents the PCM parameters that we assume.

Two alternative technologies, memristor-based memory and spin-torque transfer memories are also competing to be the next persistent storage technology. These technologies are further from production, but may offer 
improvements in writing: memristor could have an order of magnitude better endurance, and spin-torque may have unlimited endurance. Both would benefit from the memory protection and consistency provided by our toolbox.

\subsection{NVRAM on the Memory Bus}

The latency of today's external buses-PCI at a few hundred nanoseconds [17] and SATA and SAS even slower-dominates that of NVRAM. In contrast, the $40 \mathrm{~ms}$ access time of Flash memory dominates bus latency. Similarly, accessing NVRAM through a file system or system call interface incurs mode switch and data copy overhead, potentially doubling the access latency.

Systems are unlikely to abandon DRAM: Because of the wear-out and slower writes of NVRAM, we assume that at least for write-intensive workloads, systems will instead use a combination of DRAM and NVRAM, harnessing the best properties of each.

As prior work did [11], we assume that NVRAM devices will provide atomic writes at some granularityhere, a cache line (64 B), because CPUs are optimized to access DRAM at this granularity.

\subsection{Hardware Wear Leveling Limitations}

There are at least two reasons why software will be required to complement hardware solutions for avoiding NVRAM wear out.

First, hardware wear leveling can reduce performance. These solutions transparently remap the physical NVRAM locations and the logical addresses exposed to the memory controller $[30,31,36]$. To do so, they must copy content between physical locations, reducing write bandwidth and increasing overall wear. This overhead is small for well-behaved workloads [30], but wear leveling must also handle high write traffic to one or a small set of locations [36]. Doing so requires data to be moved as often as once per every application write [31]. The newest adaptive schemes penalize only those applications that concentrate many writes to few locations [31]. This solution is effective for applications using NVRAM as volatile memory, because most writes with high locality can be absorbed by a large DRAM cache, but not for applications using NVRAM persistently, where NVRAM sits only behind a small CPU cache. In this case, applications that desire high performance must avoid bad write patterns.

Second, software should avoid unnecessary writes even with wear-leveling: each write reduces the memory lifetime. As we show in Section 8, existing malloc implementations, designed for DRAM, generate excessive writes when used for NVRAM.

\subsection{Operating System Support}

We expect that existing virtual memory mechanisms will be used to map both volatile and non-volatile memory into the processes address space. Processes will be able to request NVRAM pages from the operating system, using an extension to the mmap system call. We assume that the basic unit for NVRAM in operating systems will be the $4 \mathrm{~KB}$ page, with continuing CPU and operating system support for large pages for efficiency.

To restore persistent application state, a process must be able to map the same NV memory pages across machine reboots. Therefore, operating systems must provide a way to identify groups of pages under a persistent namespace. There are multiple satisfactory proposals [10, 34, 43]. We assume that (1) it will be possible to map the same NVRAM pages in different, not necessarily concurrent processes; and (2) the OS will provide access control to regions of NVRAM, perhaps akin to access control in file systems.

We do not address the concern of persistent memory pointers. There exist various solutions: making mappings fixed [43], or using pointer swizzling [10, 44]. Another simple solution could be using the CPU support for segmentation (still present on $\mathrm{x} 86$ processors): map each logical group of NVRAM pages in its own segment and make every pointer relative to that segment.

\section{Memory Allocation for NVRAM}

The NVMalloc allocator helps address two of our three challenges for using NVRAM: preventing wear-out, by minimizing writes and helping with wear-leveling, and increasing the allocator's robustness to erroneous writes.

\subsection{Wear-Aware Memory Allocation}

Not only are today's memory allocators not optimized for NVRAM, they may actually contribute to wear-out or performance degradation when used for NVRAM. Consider as a representative example GNU malloc version 2.12.1. Malloc and several other allocators [45] cache and reuse small allocations ("blocks") in LIFO order after they are freed. This concentrates writes in a few locations, which we verify experimentally in Section 8.1.1. Malloc furthermore maintains metadata in headers and footers of each allocated (or freed) memory block, containing the size of that block. This metadata is updated for every merge and split of free blocks, which can happen for any allocation or deallocation that is not small enough to be cached. As a result, simple patterns such as a sequence of allocations followed by deallocations in the same order can cause multiple writes to the first 
header and the last footer of a series of contiguous regions. Finally, these in-place metadata updates, while memory efficient, cause additional writes that increase NVRAM wear-out; we show in Section 8.1.1 that malloc may cause $50 \%$ more writes than our allocator. Note that these malloc design decisions make sense for DRAM, providing memory efficiency and performance, but they have unintended results when applied to NVRAM.

NVMalloc is a robust, wear-aware memory allocator that avoids these problems. NVMalloc is based on two ideas: (1) limit the frequency with which any particular block of memory can be allocated, and (2) maintain frequently changing metadata in DRAM, separate from the managed blocks of non-volatile memory.

Allocator wear-leveling. NVMalloc will not reallocate a block as soon as it is freed. Instead, it timestamps the block and adds it to a FIFO queue of recently freed blocks - the don't-allocate list. On every allocation or release, the allocator examines the block at the head of the queue; if it has been in the queue for at least time $T$, it removes the block from the queue and marks it eligible for reallocation. Blocks will not be allocated with a frequency higher than $1 / T$. Assuming that the sizes of allocation requests are smaller than the available amount of non-volatile memory in the system, we can improve wear leveling by increasing $T$. To avoid using extra space, the don't-allocate list is implemented as a simple linked list, with the pointers and the timestamps stored inside the free memory blocks themselves.

Reducing allocator metadata writes. The first and easiest step that NVMalloc takes to reduce writes is using a minimum allocation size of 64 bytes - the minimum NVRAM write size. Smaller blocks cause write amplification because writes perturb the other bits in the write unit.

The second optimization reduces writes due to managing free space. Allocators reduce address space fragmentation by satisfying allocations from appropriately sized individual free blocks. For example, to allocate a 256 byte block, the allocator would only allocate from a new $4 \mathrm{~KB}$ page if it could not find a 256 byte "hole" in otherwise allocated regions. To accomplish this, NVMalloc, like malloc and its predecessor dlmalloc [22], uses segregated free lists - a free list for different size free blocks, with a common list for large blocks.

NVMalloc reduces the number of writes to persistent memory by observing that tracking the information needed to minimize fragmentation is an optimization, not something required for correctness. NVMalloc persistently stores the correct allocated/free state of every block, but relegates fragmentation information to DRAM, rebuilding this information if needed after a crash.

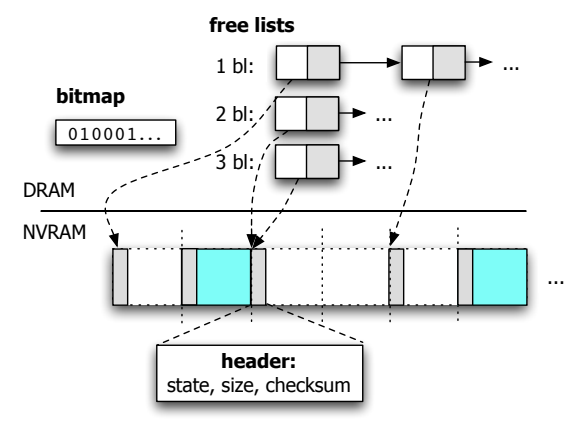

Figure 1: Memory allocator metadata example. Two of the total six basic memory blocks depicted in the diagram are allocated.

Traditional allocators store list pointers inside the headers and footers of freed blocks. When they merge adjacent free blocks into a larger free region, they update those in-place pointers. NVMalloc instead tracks the allocated/free state of each basic memory block using a DRAM bitmap (see Figure 1). To persist allocation state across reboots, each memory block has a header with its size and allocation state. When mapping a new NVRAM region, the allocator can rebuild the bitmap by scanning these headers plus the free lists described below. Unlike malloc, however, NVMalloc does not update the headers when merging free blocks, and no longer requires footers.

NVMalloc maintains its segregated free lists in DRAM. These lists may become inconsistent with the real allocation state, potentially requiring a few extra reads during allocation, but substantially reducing the number of writes. Upon freeing a block, the allocator examines the bitmap to find the maximal free region that includes that block, updates the bitmap, and adds an entry to the list corresponding to the region's size. A new allocation request is satisfied by searching through the free list with blocks of the corresponding size for the first entry that is still consistent with the information in the bitmap. If none is found, a larger free block will be split, or the allocator will request more NVRAM pages from the operating system.

NVMalloc only updates memory block headers when the block is the start of a memory region that is being allocated or when the block is freed by the application. The pointer and timestamp required for adding the block to the don't-allocate list are added in the same cache-line write as the header update (they are part of the header). As a result, NVMalloc guarantees that it will write each location at most twice per $T$ seconds.

\subsection{Robust Memory Allocation}

Bugs such as off-by-one errors, uninitialized pointers, buffer overflows or buffer underflows can corrupt mem- 
ory allocator metadata. With non-volatile memory, corrupted allocator metadata can cause permanent data loss or unrecoverable memory leaks. Consider for example a buffer underflow error that overwrites the header of an allocated memory block. When the buffer is freed, the allocator may reclaim more memory than it should, and a subsequent allocation will result in persistent application data and other block headers being overwritten, etc.

Previous approaches protect allocator metadata by storing it separately from allocated space [23, 43]. While less likely, metadata corruption could still occur (e.g., because of an uninitialized pointer that uses the data of an old stack frame corresponding to a memory allocator call). Because NVRAM corruption can lead to data loss, NVMalloc uses stronger techniques.

First, NVMalloc's design is robust to errors in its DRAM-based data structures: they only optimize allocation, their information is checked against the in-NVRAM metadata at allocation time, and they can be rebuilt if needed. Second, every NVMalloc block header contains a checksum over the size of the block, its state (allocated / free), and the position of the block relative to the beginning of the current NVRAM mapping. The latter helps detect accidental copying of whole blocks, headers included, over other blocks. The allocator can thus detect incorrect headers when allocating or freeing a block, or when scanning the block headers of a newly mapped NVRAM region. The allocator then isolates the corruption to the one or few affected blocks by scanning forward through every subsequent basic memory block (64 bytes) until it finds a new sequence of correct headers. It marks the corrupt header and notifies the application of the corruption. A side benefit of this scheme is that the allocator can also detect some erroneous writes to persistent data, something that cannot be achieved by simply separating the metadata from the data.

NVMalloc provides containment, not prevention: It limits the extent of data loss to the directly modified memory locations. It assumes that the application itself is not malicious, so the corruption is accidental. The next section presents a safety mechanism to further reduce the possibility of accidental data corruption. Our memory allocation optimizations also do not prevent memory leaks; garbage collection to ameliorate this problem is complementary to our techniques.

\section{Low-Overhead VM Protection}

For speed, applications are likely to map NVRAM storage (potentially tens or hundreds of gigabytes) into their process address spaces. Doing so, however, eliminates many traditional safety mechanisms that filesystems and databases offer. Erroneous writes by the process can corrupt not only data, but also metadata, where errors can potentially cause massive data loss. The interface through which errors can affect persistent data is now much wider: instead of writing to a buffer and invoking an explicit system call to persist it, any stray store could corrupt data. ${ }^{1}$

Concerns about the vulnerability of persistent data mapped into process address spaces date back decades, in the context of databases $[38,12]$ and reliable file system caches on battery-backed DRAM [8]. Today, OSmediated, CPU-enforced memory protection is widely used to protect parts of the address space from corruption in JVMs [18], databases [33], and garbage collection [6]. Designers of persistent data structures for NVRAM can follow this lead, but to enable them to do so, we must make this capability low-overhead enough to use in the context of hyper-fast persistent storage.

The simplest way to write-protect virtual memory on a POSIX-compliant system is to call mprotect after writing to a page. Subsequent (erroneous) writes to this page will then trigger an exception. Applications might then repair the error transparently, gracefully exit and restart, and/or simply generate a bug report noting the software bug or faulty hardware component. Unfortunately, frequent virtual memory protection changes have traditionally had a very high overhead: a page protection change involves a system call (so therefore a mode switch), acquiring locks on the page table of the process, and finally, a TLB invalidation which slows down all threads sharing the address space, not just the one issuing the system call. As a result, performance sensitive applications avoid using memory protection-e.g., database software vendors recommend turning virtual memory protection off when performance is important [42].

We improve upon the mprotect approach by (1) avoiding the system call mode switch; (2) servicing page protection requests in batches to amortize some of their costs; and (3) coalescing redundant requests such as protection and unprotecting the same page. Figure 2 depicts the high-level organization of our approach.

To protect pages, the kernel and process share a memory buffer used as a lockless producer-consumer queue [21]. The process inserts the addresses of the nonvolatile memory pages that require protection against writes, and a kernel thread periodically removes these requests from the queue to protect them. The kernel thread

\footnotetext{
${ }^{1}$ The decreasing cost of switching to kernel mode [40] makes it attractive to map persistent data in user space as readonly, and make syscalls to modify it (the data would be mapped read-write in kernel space). However, this would make application data vulnerable to stray memory accesses by device driver code. Given the large variety of third-party device drivers, we believe this would be even more difficult to control than stray accesses by the application itself.
} 


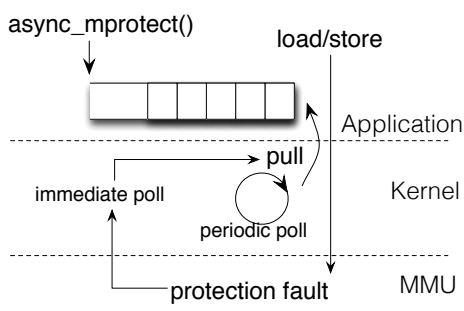

Figure 2: High-level operation of batched asynchronous memory protection.

sorts the requests and performs protection changes on ranges of pages. This approach confers two advantages:

- Changing the protection on ranges of pages and consolidating multiple requests to the same pages amortizes the cost of modifying the structures associated with the address space of the process (an expensive operation in Linux, as detailed in the next section);

- Batch processing requests means that the kernel only flushes the TLB once per batch, instead of once per protection operation, reducing slowdown imposed by frequent TLB invalidations.

This approach substantially reduces the overhead of protecting pages from writes. ${ }^{2}$ Batched asynchronous protection does not hinder the normal operation of an application because write-protecting a page does not need to happen immediately (at least not in the common case, where erroneous writes are the exception, not the rule).

Un-protecting pages that the application needs to write can follow one of two different paths, depending on the semantics that the application requires:

1) Applications, such as garbage collectors, that can predict beforehand where they will write can issue asynchronous unprotect requests early. The same approach used for protecting is used to un-protect pages before the application writes: the application puts a request in the same queue used for protect requests. When the kernel asynchronously processes a batch of requests, it first sorts the requests using a stable sorting algorithm-so that relative ordering of protect and unprotect requests for the same page is preserved. Finally, the kernel coalesces requests for the same page and performs the most recent protection change for each page in the queue.

Most applications, however, cannot predict far enough in advance which memory regions they will update. For those applications we use the second approach.

\footnotetext{
${ }^{2}$ Some potential applications require a notification queue to tell the process when the protection request was complete. This is a simple extension, but we have not yet implemented it. Applications can, however, wait for all the pending requests to be processed.
}

2) Applications that cannot foresee which page they will have to update next, use a separate unprotect queue where they write the page address/addresses of the memory object they are about to modify. They then access those pages, optimistically assuming that the page is already writable (or has become writable because of a previous asynchronous request). If the page is indeed writable, the access succeeds. If not, and the page is still protected (unwritable), the MMU generates a page fault exception.

The kernel exception handler checks the special unprotect queue, finds the unprotect request and changes the protection of the page, transparently to the application. Putting the exception handler in the kernel saves additional mode switches. This mechanism works well for applications with some access locality: a protect followed soon by an unprotect will result in no protection changes or page faults. For erroneous writes, the kernel exception handler will not find any unprotect requests, and will send the appropriate signal to the application generating that write.

\section{Cache-Efficient Updates to Persistent Main Memory}

Caching has been the primary way of bridging the gap between the speed of the CPU and that of the memory. Making an application run faster often comes down to improving its use of the caches. We expect the CPU caches to continue to play a critical role for main memory, volatile and non-volatile alike. Unfortunately, maintaining consistency in the face of crashes or power failures becomes more difficult when accessing persistent main memory through CPU caches that themselves control the order of writes to memory.

Continuously flushing cache lines introduces high overhead (e.g., more than $4 \times$ - see Section 8.3). On the other hand, bypassing the caches altogether is efficient only for those applications that write only once to a location and do not read after write (as counterexamples, consider frequent updates to a global counter-e.g., the global version of a multi-versioned data structure - or inserting ranges of keys into a $\mathrm{B}$ tree), and presents additional concerns with respect to the wear-out and write bandwidth limitations of NV memories. Furthermore, modern processors and memory controllers have been highly optimized for working with the CPU caches. We would like to use the CPU caches as normal, without flushing, but still guarantee update consistency.

The solution that we propose follows logically from our goal: make applications aware of the state of their writes in the cache so that they can make the appropriate adjustments in software. For this, we require hard- 


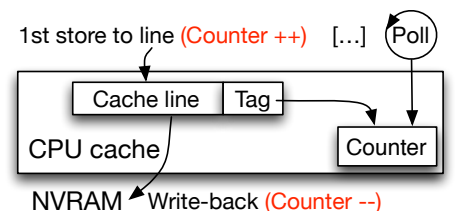

Figure 3: High-level cache line counters overview.

ware support. Given a logical group of updates, an application can check whether all the updates belonging to that group have been written out to memory-as a result of normal cache line replacement - or if some are still in the caches. Applications can use this capability in many implementations of failure atomicity: when performing shadowing, the software application will replace the original pointer with a pointer to the shadow copy after all the updates to the shadow have reached memory; when working with multi-versioned data structures, the application will increment the global version number only after the new version has been completely flushed out of the CPU caches; when updating a log, it will increment the log size only after the new record has entirely reached persistent memory, etc.

\subsection{Cache Line Counters}

To allow applications to check whether their writes have been flushed from cache, we propose light-weight hardware changes: The CPU caches are augmented with a set of counters, where each counter keeps track of how many cache lines dirtied during one atomic logical update have yet to be written back to NVRAM. An application marks the beginning of a logical update group by calling a special sgroup instruction, which tells the CPU to (1) choose a free counter, and (2) increment that counter whenever subsequent store instructions dirty new cache lines (an sgroup instruction must therefore also order stores to memory, just like the $\mathrm{x} 86 \mathrm{~s}$ fence instruction). The high-level functionality of cache line counters is illustrated in Figure 3.

A counter is incremented automatically when a store dirties a cache line, and decremented when a cache line tagged with its ID is cleaned (either by normal writeback during cache line replacement, or as a result of a clflush call). A subsequent sgroup call ends the current update group.

Applications retrieve the counter ID from a CPU register and use it later with the scheck instruction to poll the value of the counter. When scheck returns zero, the corresponding logical update group can be considered safe in NVRAM, and the counter is internally marked as free. Minor OS modifications are required to save and restore the register containing the current counter ID when a thread is preempted and re-scheduled for execution.
A store to a cache line tagged with the ID of a counter other than the current counter forces that cache line to be flushed (and the previous counter decremented) and then tagged with the ID of the current counter. This behavior is safe when multiple transactions (i.e., update groups) access the same cache lines concurrently, but may cause unnecessary cache line flushes. For improved performance, it can be changed such that a store to a cache line tagged with the ID of a counter other than the current counter does not force flushes and does not modify any counter values. However, this puts the burden to ensure safety on software: applications must track transactions that update the same cache lines concurrently and either commit them atomically (i.e., either all are committed, or none is), or in a serializable order. The latter is straightforward for applications that already serialize transactions: applications simply commit transactions in the order they were started (indifferent of the order their updates finished reaching NVRAM).

In conclusion, the interface to the cache line counter functionality consists of two new instructions (sgroup and scheck), and a new CPU register to read/write the counter ID for the current logical update group.

Unlike previous proposals for hardware support for NVRAM (the BPFS epoch barriers mechanism [11]), the modifications that we propose do not necessitate changes to the cache line replacement algorithm. This is important because forcing cache line flushes goes against the trend of enhancing the behavior of the CPU cache hierarchy with complex heuristics. Section 9 contains a more detailed comparison with BPFS.

One important implementation decision is how many counters to use. At the extreme, each cache line could be associated with a different counter, which would require as many counters as there are cache lines in the CPU caches. Die space limitations could make so many counters prohibitive. Provisioning fewer counters risks running out of counters if many applications perform many small atomic groups of updates. To solve this problem, we add a special virtual counter (we will call it $\mathrm{C} 0$ ) whose value is always zero. When there are no more free counters, the $\mathrm{CPU}$ will use $\mathrm{C} 0$. A store when $\mathrm{C} 0$ is the current counter will behave like a non-temporal write that bypasses the CPU caches. Running out of counters may affect performance, but not correctness. ${ }^{3}$

\subsection{Implementing Cache Line Counters}

Augmenting CPU caches has previously been proposed for improving cache performance [20], implementing

\footnotetext{
${ }^{3}$ This scheme has the drawback that one application might intentionally or unintentionally monopolize all the counters. Note, however, that the same is true for the CPU caches themselves.
} 
transactional memory [2, 26, 32], and even for ensuring update consistency in persistent main memory [11] (i.e., the same problem we are addressing). While we have not implemented in hardware the cache line counter mechanism, we describe here one possible implementation path. Because the counters keep track of dirty cache lines, the implementation consists mainly of a straightforward bookkeeping mechanism and a few additions to the cache coherence protocol.

Cache line tags are extended with space for a counter ID. Dirtying a cache line causes the CPU to increment the current counter and annotate the cache line with its ID. Cleaning a line decrements the counter corresponding to the ID stored in the cache line's tag (the decrement is done only after the write-back is acknowledged).

For processors with inclusive shared last level caches, like the modern Intel Core $77 \mathrm{CPU}$, it is sufficient for counters to track only the lines in the last level of the cache. This design has the advantages of avoiding the high churn in smaller caches, the counter namespace is global, and the counters are only stored in the larger cache. Reading counter values will have higher latency than if counters were stored in the smaller caches, but since applications using counters are likely to be data intensive, this latency would be dwarfed by the latencies of frequent memory accesses.

On a CPU where the last level caches are not inclusive, each core maintains a separate set of counters in its L1 cache, in a direct-mapped structure. Cache tags in shared caches store both a counter ID and a core ID. If the line cleaning/dirtying happens in a level of cache other than L1, the counter decrement/increment message must be sent up to the core that owns the counter associated with that line. ${ }^{4}$ When an application reads the value of a counter maintained by a core other than the one on which it is running, that counter value is brought in through the cache subsystem, just like normal memory

\footnotetext{
${ }^{4} \mathrm{~A}$ special case for processors with non-inclusive caches occurs when a cache line is pulled into the private cache of a core (e.g., core B) other than the one that owns the counter associated with that line (e.g., core A). In this case, the line is cleaned from all the caches accessible to core A, and sent clean to core B. Thus, core A no longer keeps track of that line. Although flushing the cache line from the first core may cause overheads for applications with this access pattern, this is not a new problem, as applications already try to avoid expensive cache line "ping-pong-ing." If, however, this occurs as a result of a thread moving to a different core, the operating system notifies the application through a signal so that it can track a new counter on the new core in addition the old counter on the previous core until those lines are cleared. This mechanism introduces some awkwardness for programmers working with counters, but we don't expect this situation to be common since operating system schedulers try to maintain core affinity (and applications can even ask that this be enforced).
}

content-the counters are memory mapped read-only.

As a concrete example of a possible instantiation of cache line counters, consider a CPU like the Core i7: a quad core CPU with $32 \mathrm{~KB}$ private L1-D and L1-I caches, $256 \mathrm{~KB}$ private $\mathrm{L} 2$, and an $8 \mathrm{MB}$ inclusive L3 cache. Such a CPU could provide 8192 one byte wide counters. ${ }^{5}$ Each counter can count up to 255 cache lines, but special counters can combine the space of two or more normal counters to be able to count up to the total number of lines. When a counter reaches its limit, the CPU can either automatically upgrade it, or treat subsequent stores as non-cacheable. For 8192 counters, the cache line tags are extended by 13 bits. Overall, this amounts to a $216 \mathrm{~KB}$ space overhead $(2.28 \%)$, incurred exclusively in the L3 cache.

\section{A B+ Tree Example}

This section describes how the proposed techniques can be used to implement a modified $\mathrm{B}+$ tree data structure, including the algorithmic changes that are required. To hide their complexity, we expect that data structures such as this would be implemented in a "persistent storage" library for NVRAM, not necessarily by individual application developers. We have applied these changes to an existing main-memory $\mathrm{B}+$ tree [5].

Using NVMalloc. NVMalloc can be used as any other memory allocator, requiring no program modifications to function correctly. However, applications can take advantage of NVMalloc's wear leveling by making small adjustments. In our $\mathrm{B}+$ tree, for example, whenever a node requires splitting, we allocate two new nodes and free the original - otherwise the same memory location risks getting heavy write traffic (see the experimental results in section 8.1.1). This simple modification is also necessary for guaranteeing consistency with cache line counters, as explained below.

Using cache line counters. First, we define what consistency properties we want for our B+ tree: (1) after a crash or power failure, we want the $\mathrm{B}+$ tree to be consistent (i.e., no dangling pointers, no partially written data), and (2) the $\mathrm{B}+$ tree must be monotonic - that is, if a power failure occurred at time $t_{1}$, the recovered data would be a subset of the data that would be recovered if the failure occurred at time $t_{2}>t_{1}$. This condition means the $\mathrm{B}$ tree behaves intuitively: once data becomes "persistent" (i.e., recoverable), it cannot be lost. To improve performance (achieve a higher insert throughput), we do not require data to be made persistent right away. These consistency

\footnotetext{
${ }^{5}$ For $1 \mathrm{~KB}$ average update group sizes, this many counters would ensure that running out of counters is rare.
} 


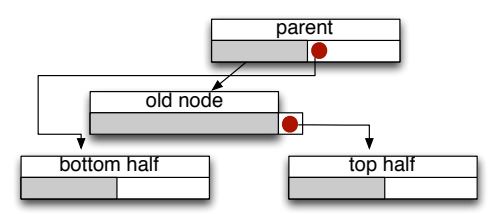

Figure 4: Transient B+-tree state after a node split. The dots mark the only pointers updated at this stage.

guarantees are reminiscent of soft updates for file systems [15].

We start by focusing on inserts. The changes that we make to the $\mathrm{B}+$ tree are to ensure consistent updates without having to flush CPU caches.

Change 1: Inserting at the end of nodes instead of keeping nodes internally sorted. In a typical B+ tree, new pointers to nodes or values are inserted into the node in sorted order, which may shift some of the existing entries. Because some entries will cross cache line boundaries, even if the algorithm is correct-it writes an entry in its new slot before overwriting the old slot-the unpredictability of cache line write-back can still cause data loss on a power failure if the update to the old slot of the entry reaches NVRAM before the update to the entry's new slot. Therefore, we append entries in log fashion, at the end of each node's list of child pointers.

A consequence of this change is that searching within a node is linear instead of a logarithmic time for a binary search, but this represents only a small penalty because nodes have few entries, and it could also be mitigated: we could maintain internally sorted read-only copies of upper-level nodes (which are read frequently and modified infrequently).

Change 2: Using cache line counters to distinguish between data that has been written completely to NVRAM and data that is not yet entirely durable. A counter tracks the cache lines dirtied by the data being written (i.e., the new inserted value). After the counter reaches zero, we mark the data as valid by writing the corresponding key in the header of the data portion (using a normal cached write) - making it easy to distinguish valid data entries from invalid ones after a power failure. $^{6}$

Change 3: Ensuring that the B+ tree is always consistent in NVRAM during node splits. Figure 4 depicts the modified node-splitting process. A node is not immediately deleted after being split. Its parent maintains its pointer to it, and the node itself is augmented with a pointer to the new node that contains the top half of its keys (the ones larger than the median key). The bottom

${ }^{6}$ No change is needed to validate the $\mathrm{B}+$ tree metadata after recovering from a crash: the keys in a valid node will match the corresponding key range in its parent. half is inserted in the parent node as usual (at the end). All subsequent inserts and searches will be performed on the new nodes. After the old keys and pointers become persistent in the new nodes, and after the pointer to the bottom half becomes persistent in the parent node (we keep track of all this using one cache line counter), the pointer to the new top half node replaces the pointer to the old node in the parent. We do not need to force this pointer update to NVRAM-because we assume cache line-sized atomic writes, a pointer will either point to the new or the old version of a node; either way, the B+ tree will be consistent by our definition. The only provision we make is to not free the old node until the pointer update has been persisted, and we can do that either with cache line counters or by relying on the delay with which our allocator reclaims space. To prevent pointer chains, if the top half is split while in the transient state, we simply replace the pointer to it with a pointer to its top half (i.e., the top half of the top half) in the old node. In summary, by keeping pointers to old versions until the new versions become persistent, we ensure we do not lose data.

For deletes, we simply invalidate the pointer (we make it NULL) to a node that is to be removed, and allow subsequent inserts to overwrite it. Moving entries from one leaf node to a neighbor and merging nodes can be handled in one of two ways: (1) lazily, when the insert load is low, by forcing cache flushes, or (2) using cache line counters (e.g., copying entries to their new node and invalidating the old one but without removing it until the entries become persistent).

Using cache line counters in implementing our $\mathrm{B}+$ tree algorithm required only small modifications to the program structure. We use a circular buffer to store "counter-action" pairs. Along with every B+ tree operation, we also check the value of the counter at the head of the buffer, and if 0 , we perform the action, which consists in updating a pointer, or writing a key to the header of a data record. If counter demand is high, we can check (and thus free) multiple counters in each round.

Using asynchronous memory protection required no modifications to the $\mathrm{B}+$ tree algorithm. We simply write the page address (or addresses) of the node (or data region) that we are about to update into the unprotect buffer, and then into the protect buffer once the update is complete.

\section{Implementation}

\subsection{NVMalloc}

NVMalloc is implemented in 735 lines of $\mathrm{C}$, and is dropin compatible with GNUMalloc. 
Allocation granularity. NVMalloc maintains a separate free list for each allocation size that is a multiple of $64 \mathrm{~B}$ (one cache line, the minimum allocation size), up to $4 \mathrm{~KB}$; recall that these lists are hints pointing to free regions of NVRAM of that size. Hints for free regions larger than $4 \mathrm{~KB}$ are placed on a common list. NVMalloc does not handle large allocations as a special case, although extending it to do so is straightforward and would reduce metadata (bitmap) overhead.

Header checksum. NVMalloc uses the CRC instruction, if available, to checksum the header. Otherwise, it XORs the block size and position into a single byte.

Failures. To ensure that allocated memory is not lost because of a power failure, a program can either (a) request that the allocator write the header of the allocated region to NVRAM before it returns, or (b) with cache line counters, simply call sgroup before making the allocation request instead of after.

\subsection{Asynchronous Memory Protection}

We implemented the async memory protection mechanism described in the previous section as a 625 line kernel module for Linux kernel version 2.6.37. Additionally, we had to make small modifications to six kernel source files (mostly for accessing functionality already in the kernel). Applications do not access the request queues directly. Instead, they use a small user-space library as the interface with the in-kernel functionality.

Batching. To control the size of request batches, we set the maximum amount of time that the kernel thread that processes requests can sleep between two subsequent batches. The actual sleep time is dynamically set to be inversely proportional to the number of new requests enqueued while a batch was being processed.

Besides coalescing requests and amortizing the cost of TLB invalidations, another benefit derived from batching is the ability to change the protection of contiguous ranges of pages instead of individual pages. In the Linux kernel, a contiguous range of pages with the same access protection is associated with a vm_area_struct structure. vm_area_struct's are chained in a doubly linked list, and also inserted into a red-black tree to ensure quick access to a structure given an address. As a consequence, changing the protection of a single page entails splitting one vm_area_struct into as many as three new structs, and then performing relatively expensive tree deletions and insertions. Furthermore, this is needless in those situations when the protection of neighboring pages would subsequently be changed anyway.

Although our implementation targeted the Linux kernel, we believe that batching memory protection change requests would benefit any operating system: any syn- chronous implementation of mprotect will have to deal with the cost of TLB invalidations and will miss the chance to coalesce multiple requests for the same page.

Lock contention. In Linux, a single lock controls access to the mm_struct of a process (the root data structure with information about the virtual address space of a process). We observed large performance penalties caused by contention for this lock: our kernel thread acquires the lock to perform page protection changes, and kernel exception handlers acquire the lock to fault-in new pages. To mitigate this slowdown, our module takes this lock only once for all the requests in a batch. Particularly performance-conscious applications can eliminate nearly all of this overhead by requesting memory from the operating system in large blocks and then touching most of the pages in advance to fault them in and prevent the cache line ping-ponging associated with different threads running on different cores contending for a lock. A more general solution would be RadixVM [9] for Linux.

\subsection{Simulating Cache Line Counters}

To understand the benefits and overheads of cache line counters, we simulate their effects in software:

Reading the cache-line counters: We simulate this in the $\mathrm{B}+$-tree implementation by maintaining a 8,192 entry table of pointers to $\mathrm{B}+$ tree nodes and values (the "virtual" counters) that the tree code reads and updates along with every insert operation. This corresponds to the application checking the value of the earliest-allocated stillactive counter and performing the associated action (i.e., copying a pointer or a key) if the counter value is 0 .

Counter allocation overhead (the sgroup instruction) is emulated using sfence, because the major overhead of sgroup is that it implies a barrier for stores.

We do not model running out of counters because applications that access gigabytes of data per second cause frequent cache line evictions, and for large groups of updates (on the order of kilobytes) the provisioned 8000 counters (see Section 5.2) are sufficient, while for smaller inserts it is easy to save counters by associating multiple consecutive inserts to one counter. Even if the counters are exhausted, the fall-back behavior (noncacheable writes) ensures correctness, as explained in Section 5.

We do not model the counter logic overhead; we believe that counter operations are simple enough that efficient implementations can hide the overhead.

\section{Evaluation}

We evaluate the techniques proposed in this paper on a desktop machine with an Intel Core i7 $8602.8 \mathrm{GHz}$ CPU, 


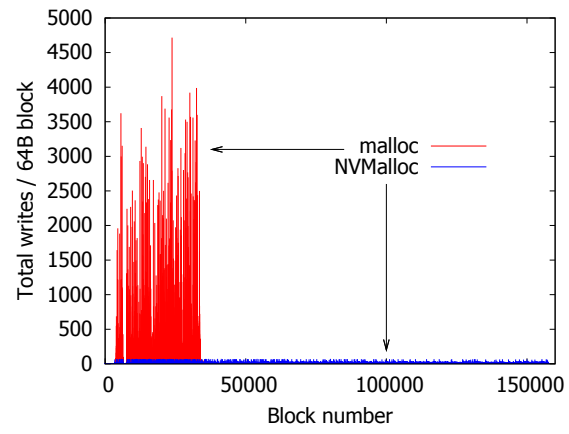

Figure 5: Writes per $64 \mathrm{~B}$ block for malloc and NVMalloc under the random alloc/free test $(50 \mathrm{~K}$ allocations and $50 \mathrm{~K}$ random deallocations with $10 \mathrm{~B}$ to $4 \mathrm{~KB}$ uniformly distributed sizes).

8 GB of DRAM memory, running Ubuntu Linux, kernel version 2.6.37, and glibc version 2.13. Since NVRAM devices for the memory bus are not yet available, we use DRAM as a proxy (Section 8.4 discusses how our results would change with NVRAM). For each performance test we report the mean (and standard error, if significant) of one hundred runs. All the applications tested run single threaded, except for the kernel thread that performs async memory protection.

\subsection{NVMalloc}

This section evaluates NVMalloc's wear leveling, fragmentation, and the overhead that it imposes on memory intensive applications.

\subsubsection{Wear Leveling}

First, we evaluate the ability of our allocator to avoid NVRAM wear-out using a simple test program that performs 100,000 random memory allocation and deallocation operations $(50 \%$ each). The sizes are uniformlydistributed between $10 \mathrm{~B}$ and $4 \mathrm{~KB}$. For every allocation, the program writes the entire allocated block once. We instrument the program using Pin [24] to record stores to memory, both when using glibc malloc and when using NVMalloc. We record the number of accesses to each 64 byte block of memory (the size of a cache line), coalescing consecutive stores to a single block (Figure 5). These stores approximate writes to NVRAM.

NVMalloc distributes writes much more evenly than (glibc) malloc. Increasing the time blocks spend on the don't-allocate list spreads the writes further. The current implementation sets this value to roughly $100 \mathrm{~ms}$ of real time; when running under Pin, we approximate this target range by waiting 2 seconds of real time to compensate for the overhead of using binary instrumentation.

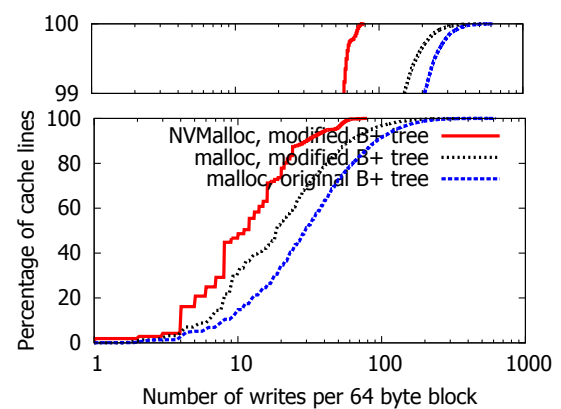

Figure 6: CDF of writes per block in B+ tree metadata, for $10^{6}$ inserts (8 B keys). Original is the classic $\mathrm{B}+$ tree implementation, modified uses NVMalloc and cache line counters. The top graph expands the $99 \%$ $100 \%$ interval. The $X$ axis is log-scale.

Without hardware wear leveling, NVMalloc's improved distribution is critical for avoiding wear-out. With hardware wear leveling, malloc's concentrated writes may require more internal remapping in the NVRAM device, degrading throughput and increasing overall wear as explained in Section 3.1.

Second, we evaluate the total number of writes. Malloc performs 1.3 times more writes to NVRAM than NVMalloc (2.39 million versus 1.83 million), because malloc maintains frequently changing metadata inside free blocks. With smaller allocation sizes (10 bytes to 256 bytes), malloc performs 1.5 times more writes. Even if perfect hardware wear leveling was possible, NVMalloc would increase NVRAM lifetime by $30 \%$ to $50 \%$ for this microbenchmark.

We repeat the wear leveling test for our modified $\mathrm{B}+$ tree. Figure 6 presents the cumulative distribution of writes per cache line-sized block of memory in the metadata portion of the $\mathrm{B}+$ tree (inner nodes and leaf nodes) for one million insert operations. ${ }^{7}$

Finally, we run the same experiment for Memcached [25] version 1.4.7, for a workload composed of $60 \mathrm{~K}$ inserts and $40 \mathrm{~K}$ random deletes-10 B keys and 256 B values. We compare NVMalloc, glibc malloc, and two other popular allocators: jemalloc [14] and Hoard [4]. The results are presented in Figure 7. NVMalloc causes orders of magnitude fewer writes to the most accessed lines than the other allocators. Overall, glibc malloc causes $8 \%$ more writes than NVMalloc, jemalloc $5 \%$, and Hoard $25 \%$.

\subsubsection{Fragmentation and Overhead}

To compare the address space fragmentation of NVMalloc and malloc, we perform one million allocations and

\footnotetext{
${ }^{7}$ This is a realistic scenario, since most allocators perform large allocations in a separate part of the address space than small allocations (for speed and fragmentation considerations).
} 


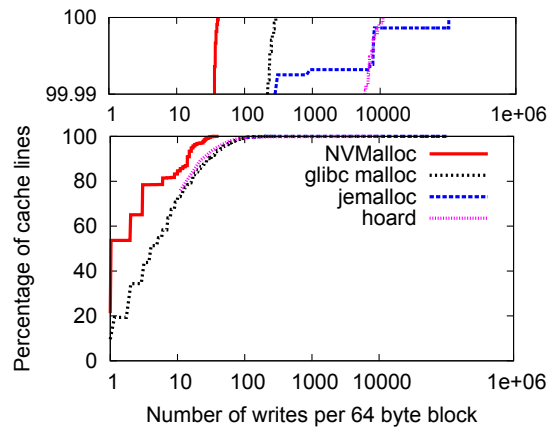

Figure 7: CDF of writes per block in Memcached (60K puts, 40K deletes, 10 B keys with 256 B values). The top graph expands the $99.99 \%-100 \%$ interval. The $X$ axis is log-scale.

\begin{tabular}{cccc}
$\begin{array}{c}\text { Free:Alloc } \\
\text { Ratio }\end{array}$ & $\begin{array}{c}\text { malloc frag. } \\
\text { total (external) }\end{array}$ & $\begin{array}{c}\text { NVMalloc frag. } \\
\text { total (external) }\end{array}$ & $\begin{array}{c}\text { Slow- } \\
\text { down }\end{array}$ \\
\hline $1 / 3$ & $1.10 \%(0.33 \%)$ & $2.14 \%(0.26 \%)$ & 1.10 \\
$1 / 2$ & $1.30 \%(0.52 \%)$ & $2.29 \%(0.41 \%)$ & 1.12 \\
$1 / 1$ & $14.34 \%(13.61 \%)$ & $12.76 \%(11.11 \%)$ & 1.10
\end{tabular}

Table 2: Fragmentation and slowdown for $10^{6}$ operations.

deallocations (in various ratios) of random, uniformly distributed sizes between $10 \mathrm{~B}$ and $4 \mathrm{~KB}$, recording the total and external fragmentation (see Table 2). The results confirm that NVMalloc produces fragmentation comparable to glibc malloc.

Table 2 also shows the slowdown that NVMalloc imposes relative to malloc. Malloc is faster for several reasons: most importantly, NVMalloc cannot cache allocations-this contravenes our wear leveling goals.

For a more realistic assessment of overhead, Table 3 compares Memcached using (a) its own slab allocator, (b) malloc, and (c) NVMalloc. The experiment sends put and delete requests in a loop embedded within the Memcached code to avoid measuring RPC overhead. There are two sets of experiments: (1) requests of random, uniformly distributed value sizes between $10 \mathrm{~B}$ and $4 \mathrm{~KB}$, and (2) fixed $1 \mathrm{~KB}$ values (10 B keys in both cases). Setting the don't-allocate time to one second (from zero) influences results by at most $1.5 \%$. The testing overhead is $1.4 \%$. NVMalloc causes little time overhead for memory intensive applications.

The space overhead equals the rate of deallocations multiplied by the time deallocated blocks spend on the don't-allocate list. For example, when making $T$ one second in the Memcached experiment with $1 \mathrm{~KB}$ values, the space overhead is $162 \mathrm{MB}$ (considering that approximately $20 \%$ of requests are misses and prompt deallocations).

\begin{tabular}{lcc} 
Allocator & Allocation size & Avg. time (std. err.) [ms] \\
\hline Memcached slab & $10 \mathrm{~B}-4 \mathrm{~KB}$ & $1768(5)$ \\
malloc & $10 \mathrm{~B}-4 \mathrm{~KB}$ & $1956(9)$ \\
NVMalloc & $10 \mathrm{~B}-4 \mathrm{~KB}$ & $1883(5)$ \\
\hline Memcached slab & $1 \mathrm{~KB}$ & $1149(4)$ \\
malloc & $1 \mathrm{~KB}$ & $1278(7)$ \\
NVMalloc & $1 \mathrm{~KB}$ & $1289(5)$
\end{tabular}

Table 3: Time for $10^{6}$ Memcached operations (80\% puts, $20 \%$ deletes).

\subsection{Asynchronous Memory Protection}

We measure the performance benefits of using async memory protection vs. synchronous mprotect.

Figure 8 shows the time to perform one million random insert operations in the in-memory $\mathrm{B}+$ tree and Memcached version 1.4.7 using async memory protection, no memory protection, and synchronous memory protection respectively. In the async case, we make an unprotect request right before we start an update to a memory object $(\mathrm{B}+$ tree node or value slot, or Memcached value item), and make a protect request as soon as we are done updating it. For the synchronous mprotect, we also maintain a user-space bitmap that records the state of a page (protected/unprotected) to avoid superfluous syscalls. We further modified Memcached to perform all operations in a tight loop, so that we discount RPC overhead. Overall application speedup with async mprotect instead of regular mprotect varies between 34\% and $500 \%$ depending on memory object sizes-async mprotect performs better for small objects because more objects will occupy the same pages, so our batching approach is able to coalesce more requests into fewer page protection changes. The overhead of async mprotect over no mprotect varies between $67 \%$ and $323 \%$.

These workloads were selected to stress the protection path in order to evaluate the speedup for protection requests conferred by the async mprotect mechanism. Real-world uses would likely incur a larger percentage of read operations, which do not require protection changes. The actual (workload-dependent) application throughput can therefore be determined from these results by weighting the overhead by the percentage of reads: For example, memcache-256B with 50\% reads would observe a roughly $2.5 \mathrm{x}$ slowdown with normal mprotect, and $1.3 \mathrm{x}$ using async mprotect.

In reducing the overhead of synchronous mprotect, sorting batched requests by page address plays an important role: without sorting, the $\mathrm{B}+$ tree test with one million $256 \mathrm{~B}$ inserts is $66 \%$ slower than with sorting.

We also measure the latency to complete a protection request from the time the application issues it until the kernel thread protects the page. Figure 9 show a CDF of 


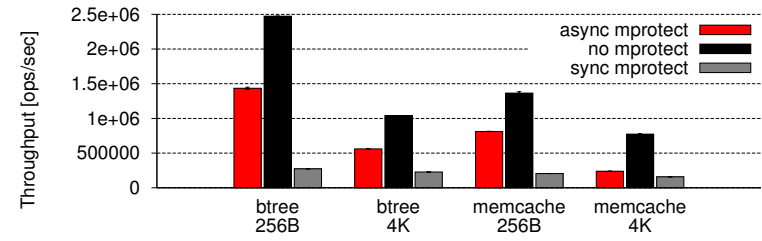

Figure 8: Operations throughput (with $95 \%$ confidence intervals) for (i) $\mathbf{B}+$ tree inserts and (ii) Memcached operations (80\% puts, $20 \%$ deletes), with $256 \mathrm{~B}$ and $4 \mathrm{~KB}$ values (8 B keys). For async mprotect, the maximum kernel thread sleep time is $150 \mathrm{~ms}$.
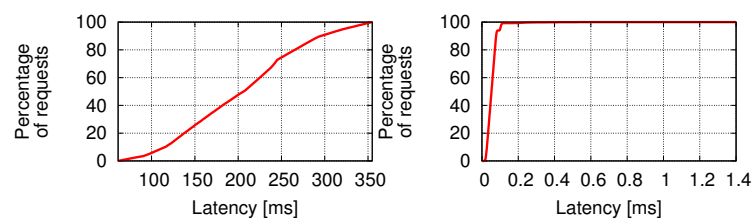

Figure 9: CDF of memory protection latency for the B+ tree using kernel thread wait times of $150 \mathrm{~ms}$ (left graph) and $0.01 \mathrm{~ms}$.

async mprotect performance with $1 \mathrm{M}$ inserts of $8 \mathrm{~B}$ keys and $256 \mathrm{~B}$ values into the $\mathrm{B}+$ tree. With Linux high resolution timers, we can achieve low latencies ( $50 \mu s$ on average) at the expense of throughput: the slowdown of low latency asynchronous mprotect is $7.9 \times$ - up from $1.72 \times$ for the high latency async mprotect, but still smaller than that of synchronous mprotect $(9 \times)$.

In conclusion, our new memory protection mechanism gives application programmers the ability to trade the latency of protection changes (how long an object is vulnerable to erroneous writes after an update, or after updates to objects in the same VM page) for update throughput.

It is important to note that the relative overheads of memory protection (both async and traditional) will be lower for NVRAM than presented here for DRAM, because of NVRAM's slower writes.

\subsection{Cache Line Counters}

This section compares the insert throughput achieved by our modified $\mathrm{B}+$ tree and the original $\mathrm{B}+$ tree [5] with (a) no modifications and therefore no consistency guarantees, (b) cache line flushes to achieve consistency (as defined in Section 6), and (c) memory mapped so as to use the write-combining memory access protocol, which guarantees that writes go (almost) immediately to

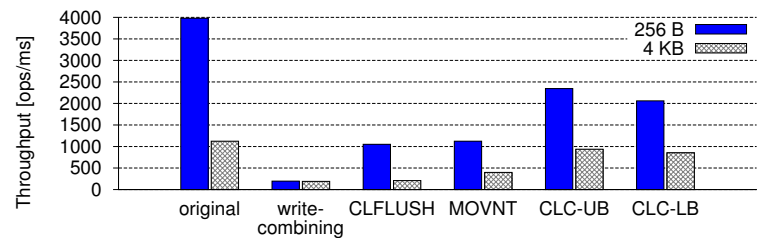

Figure 10: Insert throughput for $10^{6}$ entries (8 B keys, $256 \mathrm{~B}$ and $4 \mathrm{~KB}$ values respectively) $\mathrm{CLC}$ corresponds to cache line counters ( $\mathrm{LB} / \mathrm{UB}=$ lower/upper bound $)$.

memory. ${ }^{8}$ We also compare to the $\mathrm{B}+$ tree modified to use non-temporal streaming writes (MOVNTx Intel SSE instructions), even if they do not guarantee immediate write-back to memory (they may update only the cache if the targeted location is already in the cache [19]), to give a higher bound on what can be achieved with cachebypassing techniques. These results use the simulated cache line counter overhead (Section 7.3).

Figure 10 depicts the results. Because a node split has a transient state (see Section 6) whose duration depends on the cache line replacement rate, we present the results for the cache line counter enabled $\mathrm{B}+$ tree as two data series: one corresponding to transient states ending immediately (an upper bound for performance), and one for transient states lasting indefinitely (a lower bound).

Cache line counters outperform the other consistency preserving mechanisms for inserts. However, this comes at the expense of the read throughput: unlike the $\mathrm{B}+$ tree versions using cache flushes and cache bypassing writes, the nodes in our B+ tree do not maintain the keys in order. As a result, the read throughput decreases by $30 \%$ to $32 \%$ for $256 \mathrm{~B}$ values, and by $10 \%$ to $12 \%$ for $4 \mathrm{~KB}$ values. A possible solution is to lazily sort nodes during periods of low update load, but we leave this for future work.

\subsection{DRAM vs. NVRAM}

Our evaluation used DRAM as a proxy for NVRAM. As discussed in Section 2, some of the characteristics of DRAM are overly optimistic predictions for NVRAM. We believe, nevertheless, that "high-order bits" of our results would remain when using NVRAM, were it available: VM page tables would still be maintained in DRAM, so the mprotect overhead would be the same (in absolute value; the relative overhead will be lower, due to NVRAM's slower writes). NVMalloc's write patterns would remain the same, but, if NVRAM writes were

\footnotetext{
${ }^{8}$ Linux lacks user-space Page Attributes Table support. We ran the experiments in this section on Windows Vista 64, using Visual C++ 2010, on the same Core i7 machine, except for the tests with SSE streaming writes, which we have only been able to implement with the GCC compiler. The results of those tests that ran on both Windows and Linux were very similar.
} 
appreciably slower than DRAM writes, NVMalloc's decreased overall write traffic would give it a performance advantage over traditional allocators. Finally, the advantages of cache line counters would be more evident on NVRAM: by not forcing writes to go directly to memory, we decrease the number of memory bus transactions (which are likely to be slower with NVRAM), as well as the used write bandwidth.

\section{Related Work}

Related work falls into three main categories: systems that use NVRAM as persistent storage on the memory bus, memory management, and virtual memory protection.

NVRAM on the memory bus. Recent work has addressed some of the challenges of using NVRAM as persistent storage on the memory bus. BPFS [11] is a file system designed specifically for NVRAM. As we do, BPFS identifies cache-bypassing writes and cache flushes as an important source of inefficiency, and proposes CPU modifications - epoch barriers - to allow applications to use normal stores while getting strict guarantees for the order in which these stores will be persisted. Our cache line counter mechanism is a more general and flexible solution: applications themselves control the ordering of updates by delaying making those updates that depend on something still in the CPU caches. This allows for a less intrusive hardware implementation when compared to epoch barriers: the cache line replacement logic does not change (which is important, since otherwise this could negate the benefits of many years of CPU cache optimizations), and the CPU never has to perform cache walks when a cache line has to be written back to memory. Moreover, it is unclear if epoch barriers can work well in a general multi-process setting, outside of BPFS, because barriers would impose false dependencies between unrelated processes.

Since they do not rely on the correct functioning of external subsystems, cache line counters are more reliable than approaches that use capacitors or the residual energy in power supplies [27] to flush the CPU caches in case of power failure.

NV-heaps [10] and Mnemosyne [43] extend the software transactional memory semantics to include persistence. NV-heaps restrict the interface to NVRAM to an object-oriented programming model, and provide garbage collection for persistent memory objects-this is important, because memory leaks are especially problematic for non-volatile memory. We believe the techniques we presented in this paper are complementary to the ones introduced by NV-heaps. Mnemosyne, like Rio Vista [23] before it, also identifies the need for sep- arating the memory allocator metadata from the allocated blocks of non-volatile memory, but it stores this metadata unguarded in NVRAM - this scheme is therefore more vulnerable to corruption and data loss than NVMalloc (see discussion in Section 3.2). NV-heaps uses the BPFS epoch barriers for guarantees about ordering, while Mnemosyne relies on non-cacheable writes.

A multi-versioned $\mathrm{B}+$ tree for NVRAM has been presented in [41]. It relies on cache line flushes for maintaining write ordering - we therefore believe that it could benefit from our cache line counters scheme.

The aforementioned systems rely solely on external solutions for wear-out prevention.

Memory Management. Throughout this paper, we extensively compare NVMalloc with the GNU C Library's popular malloc implementation, which is based on dlmalloc [22]. Many other memory allocators exist [4, 14, $16]$, but they are generally focused on multithread performance (Section 8.1.1 also compares with jemalloc and Hoard). Some of their techniques work against our wear leveling goals (e.g., concentrating allocations into as few pages as possible), but most are optimizations orthogonal to ours.

Dhiman et al. [13] presented an OS-level page management technique for wear leveling page allocations. By contrast, NVMalloc is a general-purpose allocator.

Virtual Memory Protection Protecting data mapped in the address spaces of processes using virtual memory protection has been employed successfully for over two decades in the context of databases $[38,12]$ or reliable file system caches on battery-backed DRAM [8]. Mprotect continues to be used for the same purpose today in mainstream databases, but the vendors recommend turning it off when performance is important [42].

We improve the performance of this technique by making the protect operations asynchronous. Our implementation, by avoiding system calls, is reminiscent of FlexSC's exception-less system calls [37]. However, our approach gains more from being tailored to the particularities of mprotect than from avoiding mode switches.

Protecting the memory of modules running within the same address space, particularly device drivers in an OS kernel, has been the subject of much research. Nooks [39] statically provides each kernel extension with its separate page table (with write rights only for its own memory), and uses the regular hardware-enforced virtual memory protection mechanisms. Nooks does not permit direct cross-domain memory access, instead using crossdomain procedure calls. This provides stronger protection than our approach, but has lower performance when accessing memory directly. For reasons similar to ours, Nooks uses batching to mitigate the cost of changing protection domains (it batches cross-domain procedure 
calls). BGI [7] uses a compiler plug-in to generate instrumented code that checks permissions for every write to memory, at byte granularity. Unlike BGI, mprotectbased approaches work with existing, un-instrumented library code. AddressSanitizer [35] works similarly to BGI in detecting out-of-bounds accesses in user space, and we believe it could be extended to detect accidental overwrites. It has high space overhead $(3.4 \mathrm{x})$, but offers a higher level of protection than our scheme.

\section{Conclusions}

Upcoming memory technologies will soon provide a new way for applications to store persistent data at nearDRAM speeds. Harnessing this speed, however, will require placing NVRAM directly on the memory bus. Applications, supported by user-space libraries and the OS, must ensure that this persistent data remains safe from wear-out and corruption. We believe that the techniques described in this paper-a new memory allocator for NVRAM, a virtual memory protection scheme, and cache line counters-can substantially ease the task of creating safe, high-performance persistent data structures for emerging non-volatile memories.

\section{Acknowledgments}

We thank our shepherd Gernot Heiser, the anonymous reviewers, Hans Boehm, Terence Kelly, Dejan Milojicic, Dhruva Chakrabarti, Garth Gibson and Todd Mowry for their comments and suggestions. Reinhard Munz helped us with the performance evaluation. This research was funded in part by Intel via the Intel Science and Technology Center for Cloud Computing (ISTC-CC), by the National Science Foundation under award CCF-0964474, and by gifts from HP and Google.

\section{References}

[1] S. Ahn, Y. Song, C. Jeong, J. Shin, Y. Fai, Y. Hwang, S. Lee, K. Ryoo, S. Lee, J. Park, H. Horii, Y. Ha, J. Yi, B. Kuh, G. Koh, G. Jeong, H. Jeong, K. Kim, and B. Ryu. Highly manufacturable high density phase change memory of $64 \mathrm{Mb}$ and beyond. In Electron Devices Meeting, 2004. IEDM Technical Digest. IEEE International, pages 907-910, Dec. 2004.

[2] C. S. Ananian, K. Asanovic, B. C. Kuszmaul, C. E. Leiserson, and S. Lie. Unbounded transactional memory. IEEE Micro, 26:59-69, Jan. 2006.

[3] F. Bedeschi, C. Resta, O. Khouri, E. Buda, L. Costa, M. Ferraro, F. Pellizzer, F. Ottogalli,
A. Pirovano, M. Tosi, R. Bez, R. Gastaldi, and G. Casagrande. An $8 \mathrm{Mb}$ demonstrator for highdensity $1.8 \mathrm{~V}$ phase change memories. In VLSI Circuits, 2004. Digest of Technical Papers. 2004 Symposium on, pages 442-445, June 2004.

[4] E. D. Berger, K. S. McKinley, R. D. Blumofe, and P. R. Wilson. Hoard: A scalable memory allocator for multithreaded applications. In ASPLOS, pages 117-128, 2000.

[5] T. Bingmann. Stx $b+$ tree $c++$ template classes. http://idlebox.net/2007/ stx-btree/, 2008.

[6] H.-J. Boehm. The Boehm-Demers-Weiser conservative garbage collector. http: //www.research.ibm.com/ismm04/ slides/boehm-tutorial .ppt, 2004.

[7] M. Castro, M. Costa, J.-P. Martin, M. Peinado, P. Akritidis, A. Donnelly, P. Barham, and R. Black. Fast byte-granularity software fault isolation. In Proc. 22nd ACM Symposium on Operating Systems Principles (SOSP), pages 45-58, Oct. 2009.

[8] P. M. Chen, W. T. Ng, S. Chandra, C. Aycock, G. Rajamani, and D. Lowell. The Rio file cache: Surviving operating system crashes. In Proc. 7th International Conf. on Architectural Support for Programming Languages and Operating Systems (ASPLOS), pages 74-83, Oct. 1996.

[9] A. T. Clements, M. F. Kaashoek, and N. Zeldovich. Radixvm: scalable address spaces for multithreaded applications. In Proceedings of the 8th ACM European Conference on Computer Systems, EuroSys '13, pages 211-224, 2013.

[10] J. Coburn, A. M. Caulfield, A. Akel, L. M. Grupp, R. K. Gupta, R. Jhala, and S. Swanson. NV-Heaps: Making persistent objects fast and safe with nextgeneration, non-volatile memories. In Proc. 16th International Conf. on Architectural Support for Programming Languages and Operating Systems (ASPLOS), Mar. 2011.

[11] J. Condit, E. B. Nightingale, C. Frost, E. Ipek, B. Lee, D. Burger, and D. Coetzee. Better I/O through byte-addressable, persistent memory. In Proc. 22nd ACM Symposium on Operating Systems Principles (SOSP), pages 133-146, Oct. 2009.

[12] G. Copeland, T. Keller, R. Krishnamurthy, and M. Smith. The case for safe RAM. In Proceedings of the 15th International Conference on Very Large Data Bases, VLDB '89, pages 327-335. Morgan Kaufmann Publishers Inc., 1989.

[13] G. Dhiman, R. Ayoub, and T. Rosing. PDRAM: A Hybrid PRAM and DRAM Main Memory System. In Design Automation Conference, 2009. DAC '09. 
46th ACM/IEEE, pages 664 -669, July 2009.

[14] J. Evans. A Scalable Concurrent malloc(3) Implementation for FreeBSD. BSDCan - The BSD Conference, 2006.

[15] G. R. Ganger and Y. N. Patt. Soft updates: a solution to the metadata update problem in file systems. ACM Transactions on Computer Systems, 18:127153, 2000.

[16] S. Ghemawat and P. Menage. Tcmalloc : Threadcaching malloc. http://goog-perftools. sourceforge. net/doc/tcmalloc. html.

[17] B. Holden. Latency comparison between HyperTransport $^{\mathrm{TM}}$ and PCI-Express ${ }^{\mathrm{TM}}$ in communications systems. http: //www.hypertransport.org/docs / wp/Low_Latency_Final.pdf, 2006.

[18] IBM websphere real time for real time Linux, version 2 information center. http:// publib.boulder.ibm. com/infocenter/ realtime/v2ro/index.jsp, 2006.

[19] Intel 64 and IA-32 architectures developer's manual: Vol. 1. http://www. intel.com/content/www/us/en/ architecture-and-technology/, 2011.

[20] M. Kharbutli and Y. Solihin. Counter-based cache replacement and bypassing algorithms. Computers, IEEE Transactions on, 57(4):433-447, Apr. 2008.

[21] L. Lamport. Proving the correctness of multiprocess programs. Software Engineering, IEEE Transactions on, SE-3(2):125 - 143, Mar. 1977.

[22] D. Lea. A memory allocator. http://g . oswego.edu/dl/html/malloc.html, 2000.

[23] D. E. Lowell and P. M. Chen. Free transactions with Rio Vista. In Proceedings of the sixteenth ACM Symposium on Operating Systems Principles, SOSP '97, pages 92-101. ACM, 1997.

[24] C.-K. Luk, R. Cohn, R. Muth, H. Patil, A. Klauser, G. Lowney, S. Wallace, V. Janapa, and R. K. Hazelwood. Pin: Building customized program analysis tools with dynamic instrumentation. In In Programming Language Design and Implementation, pages 190-200. ACM Press, 2005.

[25] A distributed memory object caching system. http: / /memcached.org/, 2011.

[26] K. E. Moore, J. Bobba, M. J. Moravan, M. D. Hill, and D. A. Wood. LogTM: log-based transactional memory. In High-Performance Computer Architecture, 2006. The Twelfth International Symposium on, pages 254-265, Feb. 2006.

[27] D. Narayanan and O. Hodson. Whole-system persistence. In Proceedings of the seventeenth interna- tional conference on Architectural Support for Programming Languages and Operating Systems, ASPLOS '12, pages 401-410. ACM, 2012.

[28] Numonyx. Phase change memory (PCM): A new memory technology to enable new memory usage models. http://www. numonyx. com/Documents / WhitePapers / Numonyx_ PhaseChangeMemory_WhitePaper.pdf, 2009.

[29] Numonyx. Phase change memory. http: / / www.pdl.cmu.edu/SDI/2009/slides/ Numonyx.pdf, 2009.

[30] M. K. Qureshi, J. Karidis, M. Franceschini, V. Srinivasan, L. Lastras, and B. Abali. Enhancing lifetime and security of pcm-based main memory with start-gap wear leveling. In Proc. ACM MICRO, 2009.

[31] M. K. Qureshi, A. Seznec, L. Lastras, and M. Franceschini. Practical and secure pcm systems by online detection of malicious write streams. In Proc. HPCA, 2011.

[32] B. Saha, A.-R. Adl-Tabatabai, and Q. Jacobson. Architectural support for software transactional memory. In Proceedings of the 39th Annual IEEE/ACM International Symposium on Microarchitecture, MICRO 39, pages 185-196. IEEE Computer Society, 2006.

[33] Virtualized SAP performance with VMware vSphere 4. http://www.vmware.com/ files/pdf/perf_vsphere_sap.pdf, 2009.

[34] M. Satyanarayanan, H. H. Mashburn, P. Kumar, D. C. Steere, and J. J. Kistler. Lightweight recoverable virtual memory. ACM Transactions on Computer Systems, 12:33-57, Feb. 1994.

[35] K. Serebryany, D. Bruening, A. Potapenko, and D. Vyukov. Addresssanitizer: a fast address sanity checker. In Proceedings of the 2012 USENIX conference on Annual Technical Conference, USENIX ATC'12, 2012.

[36] A. Seznec. A phase change memory as a secure main memory. IEEE Comp. Arch. Letters, 9:5-8, 2010.

[37] L. Soares and M. Stumm. FlexSC: Flexible system call scheduling with exception-less system calls. In Proc. 9th USENIX OSDI, Oct. 2010.

[38] M. Sullivan and M. Stonebraker. Using write protected data structures to improve software fault tolerance in highly available database management systems. In Proc. VLDB, 1991.

[39] M. M. Swift, B. N. Bershad, and H. M. Levy. Improving the reliability of commodity operating sys- 
tems. ACM Transactions on Computer Systems, pages 77-100, 2005.

[40] How long does it take to make a context switch?

http://blog.tsunanet.net/

$2010 / 11 /$ how-long-does-

it-take-to-make-context.html.

[41] S. Venkataraman, N. Tolia, P. Ranganathan, and R. Campbell. Consistent and durable data structures for non-volatile byte-addressable memory. In Proceedings of the 9th USENIX Conference on File and Storage Technologies (FAST'11), Feb. 2011.

[42] VMware best practices for SAP installations. http://communities.vmware.com/

blogs/SAP solutions/2008/01/18/

vmware-best-practices-for

-sap-installations, 2008.

[43] H. Volos, A. J. Tack, and M. M. Swift. Mnemosyne: Lightweight persistent memory. In Proc. 16th International Conf. on Architectural Support for Programming Languages and Operating Systems (ASPLOS), Mar. 2011.

[44] P. R. Wilson. Pointer swizzling at page fault time: Efficiently supporting huge address spaces on standard hardware. SIGARCH Computer Architecture News, 19:6-13, July 1991.

[45] P. R. Wilson, M. S. Johnstone, M. Neely, and D. Boles. Dynamic storage allocation: A survey and critical review. In H. G. Baker, editor, IWMM, volume 986 of Lecture Notes in Computer Science, pages 1-116. Springer, 1995. 